\title{
EGFR methylation and outcome of patients with advanced colorectal cancer treated with cetuximab
}

\author{
ELISA CHIADINI ${ }^{1}$, EMANUELA SCARPI ${ }^{2}$, ALESSANDRO PASSARDI $^{3}$, DANIELE CALISTRI $^{1}$, \\ MARTINA VALGIUSTI $^{3}$, LUCA SARAGONI ${ }^{4}$, WAINER ZOLI ${ }^{1}$, DINO AMADORI $^{3}$ and PAOLA ULIVI ${ }^{1}$ \\ ${ }^{1}$ Biosciences Laboratory; ${ }^{2}$ Unit of Biostatistics and Clinical Trials; ${ }^{3}$ Department of Medical Oncology, \\ Istituto Scientifico Romagnolo per lo Studio e la Cura dei Tumori (IRST) IRCCS, Meldola; \\ ${ }^{4}$ Pathology Unit, Morgagni-Pierantoni Hospital, Forlì, Italy
}

Received March 28, 2014; Accepted December 1, 2014

DOI: $10.3892 / 01.2015 .2876$

\begin{abstract}
Targeted therapy of metastatic colorectal cancer $(\mathrm{mCRC})$ with monoclonal antibody anti-epidermal growth factor receptor (EGFR) agents, such as cetuximab (CTX) or panitumumab, is the treatment strategy of choice in patients characterised by a wild-type (wt) $R A S$ gene status. However, despite selection based on $R A S$ status, a high proportion of patients do not respond to therapy. EGFR methylation has been reported to have a role in predicting the response to anti-EGFR agents. The present study aimed to evaluate the role of EGFR methylation in association with the clinical outcome of patients with mCRC treated with CTX. In total, 64 patients with $\mathrm{mCRC}$ were assessed in the present study. Genomic DNA was extracted from tumoral tissue and $E G F R$ methylation and mutation of the KRAS, BRAF and PIK3CA genes were analysed by pyrosequencing. EGFR expression was assessed by immunohistochemistry. The various alterations were analysed by assessing the objective response rate (ORR), progression free survival (PFS) and overall survival (OS) rates. In total, 42 cases $(66 \%)$ exhibited $>10 \%$ EGFR methylation and there was no correlation with EGFR expression. Mean EGFR methylation of 41 and 9\% was observed in KRAS-mutated and -wt patients, respectively $(\mathrm{P}=0.05)$. Conversely, a high $E G F R$ methylation was observed in $B R A F$-wt patients with compared with patients possessing the mutated gene (18 vs. $3 \%$, respectively; $\mathrm{P}=0.07)$. EGFR methylation was significantly correlated with the OS rate [hazard ratio, $0.98 ; 95 \%$ confidence interval (CI), 0.96-1.00; $\mathrm{P}=0.019$ ], but not PFS rate. In patients with a methylation rate $<10$ and $>10 \%$, the median OS rate was 7.5 months (95\% CI, 4.4-9.4 months) and 12.0 months $(95 \%$ CI, 8.7-13.9 months), respectively $(\mathrm{P}=0.034)$. In conclusion, the
\end{abstract}

Correspondence to: Dr Paola Ulivi, Biosciences Laboratory, Istituto Scientifico Romagnolo per lo Studio e la Cura dei Tumori (IRST) IRCCS, 40 Via Maroncelli, Meldola (FC) I-47014, Italy E-mail: paola.ulivi@irst.emr.it

Key words: methylation, colorectal cancer, epidermal growth factor receptor, cetuximab present study revealed a correlation between EGFR methylation and improved OS rate in patients treated with CTX-based chemotherapy. The presence of EGFR methylation is inversely correlated with $B R A F$ and $P I K 3 C A$ mutations, indicating that the prognostic value of gene methylation may be worth verifying in further studies.

\section{Introduction}

Targeted therapy of metastatic colorectal cancer (mCRC) using monoclonal antibody (moAb) anti-epidermal growth factor receptors (EGFR) agents, such as cetuximab (CTX) or panitumumab, is the treatment strategy of choice in patients characterised by a wild type (wt) KRAS gene status (1-3). Despite the selection for $K R A S$-wt patients, only $\sim 25 \%$ of patients demonstrate a response to treatment. Several studies have attempted to identify additional potential biomarkers for the selection of patients who are likely to respond to therapy, or to exclude those who are not likely to respond (4-12). BRAF and $P I K 3 C A$ are the most frequently studied genes, and their mutations have been analysed in association with CTX response. In particular, a number of studies have demonstrated that BRAF and PIK3CA mutations were associated with a worse objective response rate (ORR), progression free survival (PFS) rate and overall survival (OS) rate, compared with wt patients, suggesting that the selection of triple-wt patients for $K R A S, B R A F$ and PIK3CA mutations may be a good strategy for improving the outcome of patients treated with CTX or panitumumab $(4,5,7-10,12)$. Although several studies have revealed a possible role of the two genes as predictive markers, others have highlighted their role as prognostic, rather than predictive, factors (13-16). NRAS mutations have also been added in clinical practice, together with $K R A S$, as a discriminating marker for moAb anti-EGFR drugs, as it has been demonstrated that patients with a mutated NRAS gene did not respond to therapy (17).

In addition, although the EGFR protein represents the target of therapy, protein expression does not appear to be a potential indicator of response (18).

A previous study has demonstrated that EGFR promoter methylation, an epigenetic event associated with the loss of EGFR expression, is associated with a poor prognosis for 
patients, highlighting the possibility that immunohistochemistry may not be the optimal method for EGFR expression assessment and that, EGFR expression may aid in predicting the effect of anti-EGFR therapies (19). However, the association between $E G F R$ methylation and the other common alterations, including $K R A S, B R A F$ and PIK3CA mutations, was not analysed.

The present study aimed to analyse the correlation between EGFR methylation and the clinical outcome of patients in a case series of mCRC patients treated with CTX-based chemotherapy. The association between EGFR methylation and $K R A S, B R A F$ and PIK3CA mutations was also analysed.

\section{Patients and methods}

Patients. In total, 64 consecutively enrolled patients with mCRC treated with a CTX-based regimen at IRST IRCCS (Meldola, Italy) between March 2004 and October 2010 were retrospectively analysed for the present study. The clinical-pathological characteristics of the patients are summarised in Table I. The inclusion criteria were a pathological diagnosis of stage IV colorectal adenocarcinoma, an age $\geq 18$ years and an Eastern Cooperative Oncology Group performance status $<3$. Patients treated prior to June 2009 were selected for CTX treatment on the basis of EGFR expression alone, as KRAS mutational status evaluation had not been made mandatory by the Italian Regulatory Authority at that time. All patients treated subsequent to June 2009 possessed tumours that were negative for KRAS mutations. This study was approved by the IRST Ethics Committee (Milan, Italy) and written informed consent was obtained from all patients.

Data were collected regarding patient characteristics, treatment and outcome. Treatment was continued until disease progression or toxicity occurred. The clinical response was assessed every eight weeks by a complete radiological examination, comprising a computed tomography (CT) or magnetic resonance imaging (MRI) scan, and it was also evaluated retrospectively according to the Response Evaluation Criteria in Solid Tumours guidelines (20). Objective tumour responses were classified into complete response, partial response, stable disease (SD) or progressive disease (PD). Patients with $\mathrm{SD}$ or PD were defined as non-responders. The objective response rate (ORR) was defined as the fraction of patients with complete or partial response confirmed $\geq 4$ weeks after the initial response. Toxicity was evaluated according to the National Cancer Institute Common Terminology Criteria for Adverse Events v3.0 guidelines (21) for each patient receiving at least one dose of CTX-based chemotherapy.

EGFR expression. The expression of EGFR was evaluated by immunohistochemistry performed on $5 \mu \mathrm{m}$-thick tissue sections obtained from paraffin-embedded tissue specimens, using the EGFR PharmaDx (Dako, Glostrup, Denmark) according to the manufacturer's instructions.

Gene mutation analyses. Formalin-fixed paraffin-embedded (FFPE) tumour blocks were reviewed for quality and tumour content. DNA was extracted from 5- $\mu \mathrm{M}$ FFPE sections of primary or metastatic lesions containing $\geq 50 \%$ tumour cells. Exon 2 of KRAS, exon 15 of $B R A F$ and exons 9 and
20 of $P I K 3 C A$ were analysed by pyrosequencing using the anti-EGFR moAb response kit for $K R A S, B R A F$ and $P I K 3 C A$ status (Diatech Pharmacogenetics, Ancona, Italy), respectively. The reactions were run on a PyroMark Q96 ID (Qiagen, Milan, Italy).

EGFR methylation analysis. EGFR methylation status was evaluated by pyrosequencing analysis. In particular, three $\mathrm{CpG}$ islands were analysed using Hs_EGFR_02_PM PyroMark CpG assays (Qiagen). The analyses were performed on PyroMark Q96 ID (Qiagen).

Statistical analysis. A two-sided Fisher's exact test was used to evaluate the association between EGFR methylation, considered to be a dichotomous variable according to a cut-off of $10 \%$, or the other gene mutations and ORR. The association between EGFR methylation, considered to be a continuous variable, and gene mutation was analysed using the Wilcoxon signed rank test.

The PFS rate was calculated from the first day of treatment to the date of the first observation of disease progression or, in the absence of progressive disease, the last follow-up or mortality. The OS rate was calculated from the first day of treatment to the date of mortality due to any cause or the date of the last follow-up. The PFS and OS rates and the $95 \%$ confidence interval (CI) were estimated using the Kaplan-Meier life-table method and the survival curves were compared using the log-rank test.

The impact of EGFR methylation on clinical outcome was evaluated in univariate analysis using a Cox regression model. All P-values were based on two-sided testing and statistical analyses were carried out using SAS statistical software (version 9.3; SAS Institute, Cary, NC, USA). P<0.05 was considered to indicate a statistically significant difference.

\section{Results}

EGFR expression. Analysis of EGFR expression was performed on 55 cases. In 34 patients, the analysis was only performed on the primary lesion, in 10 patients, only the metastatic lesion was analysed, and in 11 patients, the analysis was performed on each tumour lesion. Overall, 35 (64\%) patients exhibited positivity for EGFR expression in at least one cell. In particular, EGFR expression was evident in 29 out of $45(64 \%)$ primary tumours, and in 10 of the 21 (48\%) metastatic lesions. Concordance between EGFR expression in primary and metastatic lesions was $27 \%$, as only three cases out of 11 exhibited concordant expression. In all other cases, the primary tumour was positive for EGFR expression, whereas the metastatic lesion had lost EGFR expression.

EGFR methylation. EGFR methylation analysis was performed on three $\mathrm{CpG}$ islands in all 64 mCRC patients. For 36 patients, the analysis was only performed on the primary lesion, for 14 patients, only the metastatic lesions were analysed, and for 14 patients, analysis was possible on each lesion. Where the analysis was performed on the two lesions, the results obtained on the metastatic lesion were taken into consideration for the overall statistical analysis.

Only two cases revealed no methylation in all three $\mathrm{CpG}$ islands. Assessment of the mean methylation in the three 
Table I. Baseline patient characteristics.

\begin{tabular}{|c|c|}
\hline Characteristic & Value \\
\hline Number of patients, $n$ & 64 \\
\hline \multicolumn{2}{|l|}{ Age, years } \\
\hline Median & 61 \\
\hline Range & $34-79$ \\
\hline \multicolumn{2}{|l|}{ Gender, n (\%) } \\
\hline Male & $37(57.8)$ \\
\hline Female & $27(42.2)$ \\
\hline \multicolumn{2}{|l|}{ Performance status, n (\%) } \\
\hline 0 & $38(59.4)$ \\
\hline $1-2$ & $26(40.6)$ \\
\hline \multicolumn{2}{|l|}{ Primary tumour site, $\mathrm{n}(\%)$} \\
\hline Colon & $51(79.7)$ \\
\hline Rectum & $13(20.3)$ \\
\hline \multicolumn{2}{|l|}{ Treatment regimen, n (\%) } \\
\hline CTX+irinotecan/FOLFIRI & $57(89.1)$ \\
\hline CTX+FOLFOX4 & $6(9.4)$ \\
\hline CTX alone & $1(1.6)$ \\
\hline \multicolumn{2}{|l|}{ Previous chemotherapy, n (\%) } \\
\hline Irinotecan-based & $59(92.2)$ \\
\hline Fluoropyrimidine-based & $64(100.0)$ \\
\hline Oxaliplatin-based & $52(81.3)$ \\
\hline Bevacizumab-based & $23(35.9)$ \\
\hline \multicolumn{2}{|l|}{$\begin{array}{l}\text { Previous cancer treatments } \\
\text { for advanced disease, n }(\%)\end{array}$} \\
\hline 1 & $14(21.9)$ \\
\hline 2 & $26(40.6)$ \\
\hline 3 & $15(23.4)$ \\
\hline$>3$ & $9(14.1)$ \\
\hline \multicolumn{2}{|l|}{ Cutaneous toxicity, n (\%) } \\
\hline 0 & $16(25.0)$ \\
\hline 1 & $19(29.7)$ \\
\hline $2-3$ & $29(45.3)$ \\
\hline
\end{tabular}

FOLFIRI, folinic acid, 5-fluorouracil and irinotecan hydrochloride; FOLFOX4, folinic acid, 5-fluorouracil, and oxaliplatin.

CpG islands revealed that 22 cases (34\%) possessed a mean percentage of methylation of $<10 \%$, whereas 42 cases $(66 \%)$ exhibited $\geq 10 \%$ methylation. In particular, 40 cases $(63 \%)$ demonstrated a methylation rate between $10 \%$ and $50 \%$, whereas two cases $(3 \%)$ demonstrated an average percentage $>50 \%$ in the primary or metastatic lesions. Separate analysis of the three $\mathrm{CpG}$ islands revealed that 16 samples (25\%) exhibited a methylation level of $<10 \%$ in all three islands, 48 cases $(75 \%)$ exhibited a methylation level between 10 and $50 \%$ in at least one CpG island, and 13 cases $(20 \%)$ possessed a methylation level $>50 \%$ in one or more $\mathrm{CpG}$ islands. Of the 14 tumour samples for which the EGFR methylation analysis was performed on the primary and metastatic lesions, the results demonstrated heterogeneity between the two specimens. In particular, samples three and four clearly demonstrated an increased methylation level in the three $\mathrm{CpG}$ islands in the metastatic lesions, whereas sample six revealed a strong decrease at this site (Table II).

KRAS, BRAF and PIK3CA mutation analyses. It was found that KRAS was mutated in $19(30 \%)$ cases, comprising five G12V, three G12S, two G12D, one G12A, one G12C and seven G13D mutations. In three cases, a discordant result was obtained between the primary tumour and the metastatic lesion. In two of these cases, the primary tumour was wt and the metastatic lesion was mutated, whereas the remaining case demonstrated the opposite result, with mutation in the primary tumour and a wt metastatic lesion.

$B R A F$ was mutated in 11 cases (17.2\%). In all cases, the mutation was a V600E mutation on exon 15 of the gene. In four cases, a discordant result was observed between the primary tumour and metastatic lesion. In three of these cases, the mutation was evident in the metastatic lesion and not in the primary tumour, whereas in the remaining case, the mutation was evident in the primary tumour and not in the metastatic lesion. The $B R A F$ mutation was associated with a shorter OS (6.9 months; 95\% CI, 1.7-15.1) when compared with $B R A F$ wt patients (10.0 months; 95\% CI, 8.2-13.5) ( $\mathrm{P}=0.09)$. However, no significant differences in PFS rate were identified between the two groups.

PIK3CA was mutated in seven cases (10.9\%). In six cases the mutation was in exon 9 of the gene, consisting of one E545G, one E542K and four E545K mutations, whereas in the remaining case the mutation was in exon 20 , with a H1047L mutation. In one case, the mutation was detected in the primary tumour and not in the metastatic lesion, whereas in the other cases, a concordant result was obtained between the two lesions. The PIK3CA mutation was associated with a shorter OS (7.0 months; 95\% CI, 3.3-9.6) when comapred with PIK3CA wt patients (10.0 months; 95\%, CI 8.3-13.7) $(\mathrm{P}=0.05)$. However, no significant differences in PFS rate were identified between the two groups.

Correlation between EGFR methylation and other molecular alterations. In the primary tumour, no correlations were found between $K R A S, B R A F$ or PIK3CA mutations and EGFR methylation, which were assessed in the three $\mathrm{CpG}$ islands separately or as mean value. Conversely, in the metastatic lesions, a correlation was found between the percentage of mean EGFR methylation and the presence of KRAS mutation, or the presence of a $B R A F$-wt gene. In particular, a mean EGFR methylation of $41 \%$ was observed in $K R A S$-mutated patients, compared to a methylation level of $9 \%$ in $K R A S$-wt patients $(\mathrm{P}=0.05)$. Conversely, a high $E G F R$ methylation was observed in $B R A F$-wt patients compared with $B R A F$ mutated patients (18 vs. $3 \%$, respectively; $\mathrm{P}=0.07$ ) (Table III). No correlation was found between EGFR methylation and EGFR expression, in the primary and metastatic lesions.

EGFR methylation in association with clinical response. The mean percentage of EGFR methylation in the three $\mathrm{CpG}$ islands demonstrated no correlation with the clinical response. Conversely, a higher median value of $\mathrm{CpG}$ island 2 methylation was observed in responders $(22 \%)$ compared to non-responders $(11 \%)(\mathrm{P}=0.02)$, and the 
Table II. Methylation of three EGFR CpG islands of in the primary tumor and matched metastatic lesions.

\begin{tabular}{|c|c|c|c|c|c|c|}
\hline \multirow[b]{2}{*}{ Sample } & \multicolumn{3}{|c|}{ Methylation of primary tumor tissue } & \multicolumn{3}{|c|}{ Methylation of metastatic lesion } \\
\hline & CpG1, \% & $\mathrm{CpG} 2, \%$ & $\mathrm{CpG} 3, \%$ & $\mathrm{CpG} 1, \%$ & $\mathrm{CpG} 2, \%$ & $\mathrm{CpG} 3, \%$ \\
\hline 1 & 5 & 7 & 14 & 0 & 3 & 0 \\
\hline 2 & 7 & 55 & 15 & 18 & 32 & 74 \\
\hline 3 & 0 & 9 & 0 & 4 & 82 & 84 \\
\hline 4 & 7 & 9 & 0 & 48 & 49 & 54 \\
\hline 5 & 5 & 0 & 6 & 10 & 0 & 0 \\
\hline 6 & 65 & 61 & 63 & 0 & 4 & 0 \\
\hline 7 & 9 & 29 & 35 & 14 & 31 & 35 \\
\hline 8 & 8 & 16 & 17 & 16 & 24 & 17 \\
\hline 9 & 0 & 0 & 25 & 0 & 9 & 3 \\
\hline 10 & 2 & 27 & 36 & 0 & 5 & 4 \\
\hline 11 & 1 & 8 & 13 & 25 & 0 & 0 \\
\hline 12 & 27 & 21 & 23 & 2 & 0 & 68 \\
\hline 13 & 65 & 3 & 0 & 2 & 12 & 14 \\
\hline 14 & 17 & 21 & 32 & 40 & 61 & 33 \\
\hline
\end{tabular}

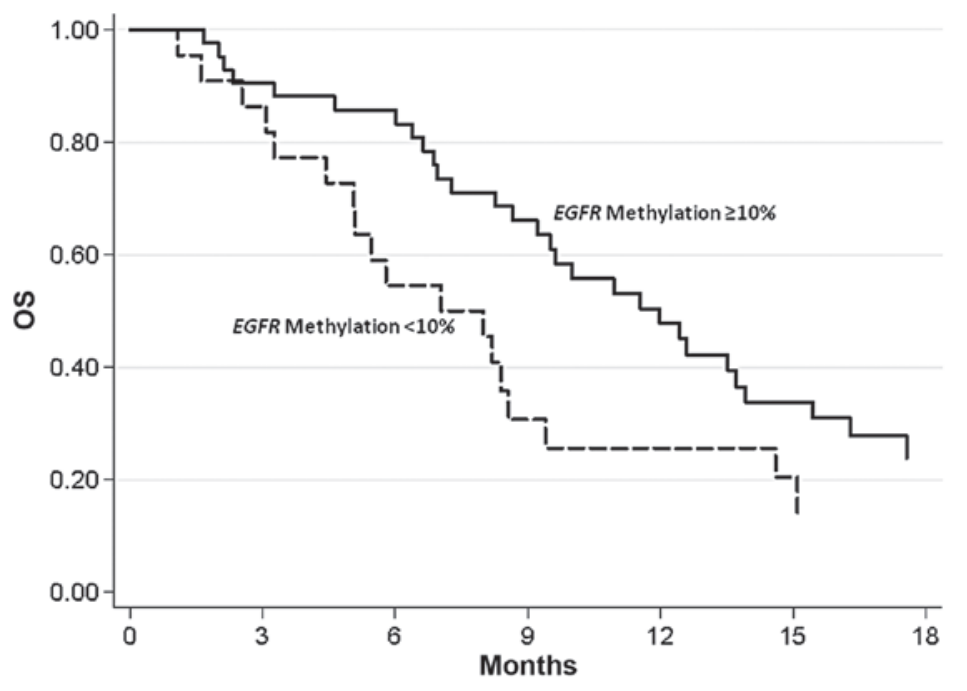

Figure 1. The association between OS rate and EGFR methylation. OS, overall survival.

univariate analysis of objective response revealed a significant correlation $(\mathrm{P}=0.037)$.

In univariate analyses, with the EGFR methylation level being considered a continuous variable, the Cox regression model revealed that EGFR methylation was significantly correlated with OS rate (hazard ratio, 0.98; 95\% CI, 0.96-1.00; $\mathrm{P}=0.019)$, but not with the PFS rate. In particular, the median OS rate of patients with a methylation rate $<10 \%$ was 7.5 months (95\% CI, 4.4-9.4 months) and the OS rate was 12.0 months (95\% CI, 8.7-13.9 months) in patients with a methylation rate $>10 \%(\mathrm{P}=0.034)$ (Fig. 1).

\section{Discussion}

Despite KRAS mutation being one of the selection criteria for the use of anti-EGFR therapy in mCRC patients, only a subgroup of $K R A S$-wt patients responded to the treatment. The analysis of other markers, including NRAS, BRAF and $P I K 3 C A$, have also allowed for the selection of further patients that may be likely to not respond to therapy $(17,22)$. With a multiple marker selection, the probability of the selected patients responding to treatment may be significantly increased (4).

Although the target of moAb anti-EGFR drugs is the EGFR protein, several studies analysing the correlation between the expression or amplification of EGFR and clinical response have reported non-significant results (23-25). However, it is also likely that immunohistochemical evaluation of the EGFR protein expression may not be sufficiently accurate to detect the loss of EGFR protein in cancer tissue, thus compromising data analysis and interpretation (26). In addition, several biases may compromise the reproducibility of immunohistochemical 


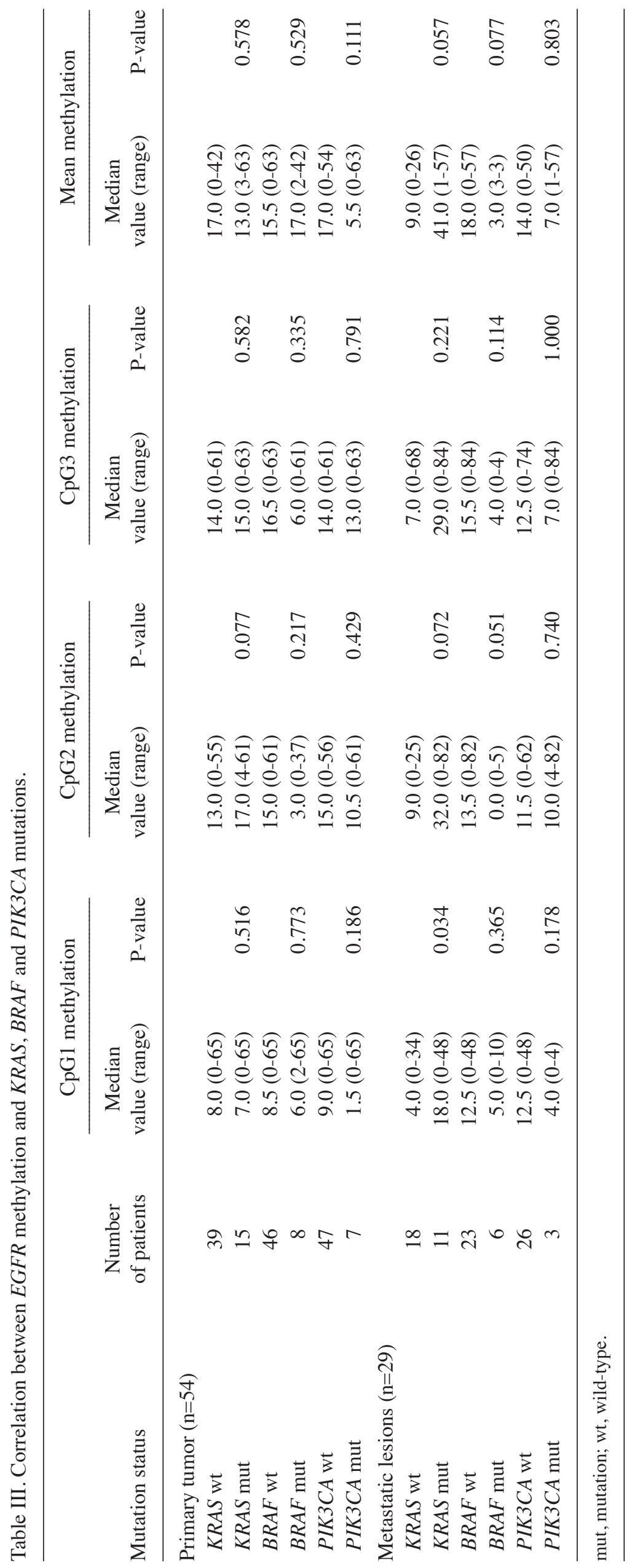


analysis, including the different antibodies used and the varying reaction conditions that are used between different laboratories.

Gene methylation analysis may be an indirect approach towards analysing gene expression. It has been demonstrated that EGFR methylation is detectable in several types of solid tumour (27), but its association with EGFR immunohistochemical expression is unclear.

A previous study has demonstrated a significant correlation between the absence of EGFR methylation and the response to CTX-based chemotherapy in colorectal cancer patients. In particular, patients with a methylated gene were less responsive to therapy compared with patients without methylation, although no correlation between EGFR methylation and expression was identified (19). Conversely, in the present study, a significant correlation was found between the methylation of specific $\mathrm{CpG}$ islands and response to treatment, demonstrating that gene methylation was significantly correlated with ORR and OS rate, but not with PFS rate. The reasons for the discrepancy between the present study and the study by Scartozzi et al (19) may lie in the different $\mathrm{CpG}$ islands analysed and the different methodologies used. In particular, the present study analysed three $\mathrm{CpG}$ islands localised in the promoter region of EGFR (27), but these were upstream of the region analysed in the study by Scartozzi et al. In addition, pyrosequencing methodology was used in the present study, whereas the methylation-specific polymerase chain reaction approach was used in the aforementioned study.

To the best of our knowledge, the present study analysed the correlation between $E G F R$ methylation and mutation in $K R A S, B R A F$ and PIK3CA, which are genes involved in the resistance mechanisms to CTX, for the first time. No significant correlation was observed overall between EGFR methylation and the various gene mutations. However, it was only in metastatic lesions that gene methylation was found to be positively correlated with KRAS mutation and negatively correlated with $B R A F$ mutation. In particular, a significantly increased percentage of methylation was observed in $K R A S$ mutated and $B R A F$-wt patients. Additionally, a high level of methylation was observed more frequently in $P I K 3 C A-w \mathrm{t}$ patients compared with $P I K 3 C A$-mutated patients, although this difference was not significant.

In the present study, the $B R A F$ and $P I K 3 C A$ mutations were correlated with a shorter OS rate, and the correlation identified between these mutations and the absence of EGFR methylation is consistent with the worse predictive value observed in patients with an EGFR methylation level $<10 \%$. The present study included patients with KRAS-wt and -mutated tumours that were treated with CTX prior to June 2009, and other patients selected for $K R A S$-wt tumours that were treated subsequent to June 2009. No significant correlations between the KRAS mutation status and the ORR, PFS rate and OS rate were identified, most likely due to the limited number of KRAS-mutated cases.

The correlations identified in metastatic lesions between EGFR methylation and KRAS mutation, may be due to the presence of constitutively activated signalling prompted by $K R A S$ inducing cells to overcome the role of an EGFR hyperexpression. The inverse association identified with $B R A F$ and PIK3CA mutations, by contrast, remains to be elucidated.
Similar to the study by Scartozzi et al (19), the present study did not identify any correlation between EGFR methylation and EGFR expression, despite the varying results obtained in terms of methylation.

In conclusion, the present study demonstrated correlations between $E G F R$ methylation and an improved response and OS rate in patients treated with CTX-based chemotherapy. The presence of EGFR methylation was found to be inversely correlated with $B R A F$ and $P I K 3 C A$ mutations, indicating that the prognostic value of gene methylation requires additional verification in future studies.

\section{Acknowledgements}

The authors would like to thank Ms. Ursula Elbling for editing the original manuscript.

\section{References}

1. Lièvre A, Bachet JB, Le Corre D, et al: KRAS mutation status is predictive of response to cetuximab therapy in colorectal cancer. Cancer Res 66: 3992-3995, 2006.

2. Benvenuti S, Sartore-Bianchi A, Di Nicolantonio F, et al: Oncogenic activation of the RAS/RAF signaling pathway impairs the response of metastatic colorectal cancers to anti-epidermal growth factor receptor antibody therapies. Cancer Res 67: 2643-2648, 2007.

3. Amado RG, Wolf M, Peeters M, et al: Wild-type KRAS is required for panitumumab efficacy in patients with metastatic colorectal cancer. J Clin Oncol 26: 1626-1634, 2008.

4. Sartore-Bianchi A, Di Nicolantonio F, Nichelatti M, et al: Multi-determinants analysis of molecular alterations for predicting clinical benefit to EGFR-targeted monoclonal antibodies in colorectal cancer. PLoS One 4: e7287, 2009.

5. Di Nicolantonio F, Martini M, Molinari F, et al: Wild-type $\mathrm{BRAF}$ is required for response to panitumumab or cetuximab in metastatic colorectal cancer. J Clin Oncol 26: 5705-5712, 2008.

6. Frattini M, Saletti P, Romagnani E, et al: PTEN loss of expression predicts cetuximab efficacy in metastatic colorectal cancer patients. Br J Cancer 97: 1139-1145, 2007.

7. Perrone F, Lampis A, Orsenigo M, et al: PI3KCA/PTEN deregulation contributes to impaired responses to cetuximab in metastatic colorectal cancer patients. Ann Oncol 20: 84-90, 2009.

8. Sartore-Bianchi A, Martini M, Molinari F, et al: PIK3CA mutations in colorectal cancer are associated with clinical resistance to EGFR-targeted monoclonal antibodies. Cancer Res 69: 1851-1857, 2009.

9. Jhawer M, Goel S, Wilson AJ, et al: PIK3CA mutation/PTEN expression status predicts response of colon cancer cells to the epidermal growth factor receptor inhibitor cetuximab. Cancer Res 68: 1953-1961, 2008.

10. Laurent-Puig P, Cayre A, Manceau G, et al: Analysis of PTEN, BRAF, and EGFR status in determining benefit from cetuximab therapy in wild-type KRAS metastatic colon cancer. J Clin Oncol 27: 5924-5930, 2009.

11. Razis E, Briasoulis E, Vrettou E, et al: Potential value of PTEN in predicting cetuximab response in colorectal cancer: An exploratory study. BMC Cancer 8: 234, 2008.

12. Ulivi P, Capelli L, Valgiusti M, et al: Predictive role of multiple gene alterations in response to cetuximab in metastatic colorectal cancer: A single center study. J Transl Med 10: 87, 2012.

13. Van Cutsem E, Köhne CH, Láng I, et al: Cetuximab plus irinotecan, fluorouracil, and leucovorin as first-line treatment for metastatic colorectal cancer: Updated analysis of overall survival according to tumor KRAS and BRAF mutation status. J Clin Oncol 29: 2011-2019, 2011.

14. Ogino S, Nosho K, Kirkner GJ, et al: $\mathrm{CpG}$ island methylator phenotype, microsatellite instability, BRAF mutation and clinical outcome in colon cancer. Gut 58: 90-96, 2009.

15. Tol J, Nagtegaal ID and Punt CJ: BRAF mutation in metastatic colorectal cancer. N Engl J Med 361: 98-99, 2009. 
16. Ogino S, Nosho K, Kirkner GJ, et al: PIK3CA mutation is associated with poor prognosis among patients with curatively resected colon cancer. J Clin Oncol 27: 1477-1484, 2009.

17. Douillard JY, Oliner KS, Siena S, Tabernero J, et al: Panitumumab-FOLFOX4 treatment and RAS mutations in colorectal cancer. N Engl J Med 369: 1023-1034, 2013.

18. Valentini AM, Pirrelli M and Caruso ML: EGFR-targeted therapy in colorectal cancer: Does immunohistochemistry deserve a role in predicting the response to cetuximab? Curr Opin Mol Ther 10: $124-131,2008$.

19. Scartozzi M, Bearzi I, Mandolesi A, et al: Epidermal growth factor receptor (EGFR) gene promoter methylation and cetuximab treatment in colorectal cancer patients. $\mathrm{Br} J$ Cancer 104: 1786-1790, 2011.

20. Therasse P, Arbuck SG, Eisenhauer EA, et al: New guidelines to evaluate the response to treatment in solid tumors. European Organization for Research and Treatment of Cancer, National Cancer Institute of the United States, National Cancer Institute of Canada. J Natl Cancer Inst 92: 205-216, 2000

21. National Cancer Institute: Cancer Therapy Evaluation Program, Common Terminology Criteria for Adverse Events, Version 3.0. http://ctep.cancer.gov. Accessed December 17, 2013.

22. De Roock W, Claes B, Bernasconi D, et al: Effects of KRAS, BRAF, NRAS, and PIK3CA mutations on the efficacy of cetuximab plus chemotherapy in chemotherapy-refractory metastatic colorectal cancer: A retrospective consortium analysis. Lancet Oncol 11: 753-762, 2010.
23. Cunningham D, Humblet $\mathrm{Y}$, Siena S, et al: Cetuximab monotherapy and cetuximab plus irinotecan in irinotecan-refractory metastatic colorectal cancer. N Engl J Med 351: 337-345, 2004

24. Scartozzi M, Bearzi I, Mandolesi A, et al: Epidermal growth factor receptor (EGFR) gene copy number (GCN) correlates with clinical activity of irinotecan-cetuximab in K-RAS wild-type colorectal cancer: A fluorescence in situ (FISH) and chromogenic in situ hybridization (CISH) analysis. BMC Cancer 9: 303, 2009.

25. Licitra L, Störkel S, Kerr KM, et al: Predictive value of epidermal growth factor receptor expression for first-line chemotherapy plus cetuximab in patients with head and neck and colorectal cancer: Analysis of data from the EXTREME and CRYSTAL studies. Eur J Cancer 49: 1161-1168, 2013

26. Atkins D, Reiffen KA, Tegtmeier CL, Winther H, Bonato MS and Störkel S: Immunohistochemical detection of EGFR in paraffin-embedded tumor tissues: Variation in staining intensity due to choice of fixative and storage time of tissue sections. $\mathrm{J}$ Histochem Cytochem 52: 893-901, 2004.

27. Montero AJ, Díaz-Montero CM, Mao L, Youssef EM, Estecio M, Shen L and Issa JP: Epigenetic inactivation of EGFR by $\mathrm{CpG}$ island hypermethylation in cancer. Cancer Biol Ther 5: 1494-1501, 2006. 